\title{
Labyrinthe
}

$8 \mid 2001$

Numéro 8

\section{Le problème de l'identité culturelle dans la tradition libérale}

\section{Claire Demesmay}

\section{(2) OpenEdition \\ 1 Journals}

Édition électronique

URL : http://journals.openedition.org/labyrinthe/867

DOI : $10.4000 /$ labyrinthe.867

ISSN : 1950-6031

Éditeur

Hermann

\section{Édition imprimée}

Date de publication : 1 janvier 2001

Pagination : 112-115

Référence électronique

Claire Demesmay, « Le problème de l'identité culturelle dans la tradition libérale », Labyrinthe [En ligne], 8| 2001, mis en ligne le 25 mai 2005, consulté le 19 avril 2019. URL : http://journals.openedition.org/ labyrinthe/867 ; DOI : 10.4000/labyrinthe.867

Ce document a été généré automatiquement le 19 avril 2019.

Propriété intellectuelle 


\title{
Le problème de l'identité culturelle dans la tradition libérale
}

\author{
Claire Demesmay
}

1 Mon travail a porté sur le débat qui est aujourd'hui au cœur de la philosophie politique, c'est-à-dire celui du libéralisme politique et de son fondement individualiste, confronté au communautarisme.

2 Afin d'éviter toute confusion, précisons que le courant libéral, qui ne correspond pas en philosophie politique au sens qu'on lui attribue ordinairement, notamment en économie, se situe sur la gauche de l'échiquier politique : la pensée libérale est attachée à la démocratie constitutionnelle et à un système égalitaire garantissant à chacun les mêmes droits et libertés, et s'élève contre l'individualisme ultralibéral et la conception minimaliste de l'État de la théorie libertarienne, telle que l'incarne aujourd'hui Nozick ${ }^{1}$. En Europe, c'est le courant social-démocrate qui lui fait le mieux écho.

3 Les sociétés libérales, c'est-à-dire les sociétés démocratiques occidentales, sont aujourd'hui marquées par un double mouvement. Dans la pensée sociologique et philosophique, ainsi que dans la vie sociale et politique, l'individualisme ne cesse de gagner en importance et en valeur; en théorie, c'est le règne de l'individu, considéré comme la valeur suprême des domaines économique, politique et moral, c'est-à-dire comme un sujet de droit et comme l'un des fondements majeurs, à la fois du fonctionnement des sociétés libérales et de la théorie libérale qui en énonce les principes. En pratique, il est incontestable que les membres de la société libérale sont toujours plus indépendants vis-à-vis des structures communautaires, qu'il s'agisse des Églises, des partis politiques ou même du cercle familial ; ils agissent de plus en plus souvent comme des êtres autonomes, libres de choisir ce qui leur convient, sans accepter de se laisser dicter leur comportement par une instance supérieure.

4 Parallèlement à ce phénomène d'atomisation du social qu'avait déjà pressenti et relevé Tocqueville au XIX ${ }^{\mathrm{e}}$ siècle, et qui révèle une société démembrée pouvant difficilement se 
référer à des valeurs communes partagées par l'ensemble de ses citoyens, se développe une affirmation publique des identités culturelles, qui prend des formes différentes mais renvoie dans tous les cas à une même revendication fondamentale : celle de la reconnaissance, par les instances publiques, d'appartenances identitaires particulières. Ici, l'individu n'est plus cet être indépendant qui crée par lui-même sa propre culture, unique par son originalité et plurielle par sa nature de multi-appartenance. Il est l'un des membres d'un groupe particulier qui constitue la clef de son être, et d'où découle le sens de son existence. Contrairement au libéralisme, le mouvement communautarien, comme l'indique son appellation, érige la communauté en valeur essentielle, et cherche à faire du groupe le fondement même de la société. Afin de retrouver la stabilité et la chaleur communautaires auxquelles ils n'ont souvent plus la possibilité d'accéder dans la société libérale, les individus sont encouragés à regagner leur groupe d'appartenance, sans lequel ils éprouveraient d'immenses difficultés à découvrir leur véritable identité et à mener une vie satisfaisante. Dans une telle problématique, la communauté est le tuteur psychologique et moral de tout homme.

5 Apparu dans les sociétés libérales peu après le développement immodéré du processus d'individualisation, le mouvement communautarien s'oppose à ce dernier en théorie comme en pratique. D'une part, ses représentants ne cessent, depuis 1971, année de parution de Théorie de la justice ${ }^{2}$, ouvrage de référence par excellence du libéralisme contemporain, de publier des textes critiques attaquant les principes libéraux et dénonçant leurs prétendues erreurs théoriques. Sur le terrain de la philosophie politique, "l'équipe $C »^{3}$, composée de Michael Sandel, Charles Taylor, Alasdair MacIntyre et Michael Walzer, reproche à « l'équipe $\mathrm{L}$ » de John Rawls, Ronald Dworkin et Thomas Nagel sa conception trop abstraite du moi, qui, d'après elle, ne tient pas compte de la réalité de la socialisation et des besoins communautaires des hommes, condamne l'écart séparant l'application de ses principes de sa théorie et dénonce les excès individualistes et atomistes de cette dernière. De l'autre, la société libérale voit l'émergence de nombreuses pressions et manifestations identitaires qui prennent des expressions différentes; entre les homosexuels qui exigent la reconnaissance publique de leur identité particulière, les populations immigrées d'Afrique du Nord affirmant que le respect de leur religion passe par le port du foulard à l'école, et les féministes qui revendiquent le droit à la parité politique, il y a ce dénominateur commun : tous se veulent les représentants d'une communauté particulière, et souhaitent être regardés et traités comme tels par l'État et par les autres citoyens.

6 L'individualisme immodéré et l'apparition de nouvelles affirmations identitaires semblent aller de pair, être indissociables l'un de l'autre ; ils paraissent même tellement liés l'un à l'autre que Walzer ${ }^{4}$ 'hésite pas à affirmer qu'ils ne sont que les deux faces d'un même phénomène à l'influence réciproque. Les revendications d'appartenance apparaissent comme la conséquence d'une puissance libérale si dévastatrice qu'elle ne doit plus être tolérée en tant que telle, et les excès atomistes du libéralisme comme les produits de ces affirmations identitaires démesurées. Ces deux mouvements semblent être deux forces contraires qui représentent pareillement un danger considérable pour la société libérale, notamment pour son lien social. Leur antagonisme crée un phénomène de répulsion qui contribue à creuser entre eux un écart toujours plus grand, à rendre leurs principes et leurs revendications de plus en plus excessifs et, finalement, à placer la société libérale au centre d'une redoutable confrontation risquant de dissoudre ses idéaux humanistes et de briser sa cohésion. Si de tels problèmes apparaissent dans les 
sociétés libérales plus qu'ailleurs, c'est bien entendu parce que celles-ci sont définies par un pluralisme culturel structurel, autrement dit parce qu'elles sont constituées de plusieurs composantes culturelles souvent très différentes les unes des autres.

7 En s'imposant sur les scènes philosophique et sociale, le communautarisme lance un défi aux tenants du libéralisme : repenser la problématique de l'identité, non plus à travers le prisme de l'individu, comme ce fut - et c'est encore - le fait de la tradition libérale, mais du groupe : l'individu, véritable fondement du libéralisme, serait une entité abstraite et quasi inutile, qui ne rend compte ni des liens d'appartenance dans la société réelle, ni des processus de constitution de l'identité culturelle, et qui aurait fortement contribué à l'érosion des communautés, laissant des citoyens tristement identiques, unis dans la cité par un lien politique fragile et froid, désarmés face à des valeurs politiques universelles mais vides. Derrière l'affirmation identitaire du communautarisme, tentant de se substituer au partage d'une identité politique universelle, se profilent trois questions majeures : la première se rapporte au problème de la différence à l'intérieur de la société libérale ; la deuxième, à la place d'une minorité par rapport à une majorité ; et la troisième, à laquelle sont subordonnées les deux précédentes, au problème de l'identité culturelle et de sa reconnaissance par les principes libéraux. Ces questions remettent en cause de nombreux principes du libéralisme, tel qu'il a été développé aux XVII ${ }^{e}$ et $\mathrm{XVIII}^{\mathrm{e}}$ siècles par Kant, Locke et Mill et tel qu'il est encore aujourd'hui pensé et appliqué. Il est bien sûr question du respect des droits de l'individu, mis en péril par les revendications de droits collectifs inégaux, accordés aux membres de la société en fonction de leur appartenance communautaire, non plus de leur simple statut de citoyens, conçus comme porteurs des mêmes droits et libertés inaliénables.

8 L'enjeu, théorique mais également social et politique, du débat fait qu'il est indispensable pour les libéraux d'écouter les revendications communautariennes, signes d'une certaine difficulté de réception de leur théorie, et de se demander quels sens et rôle peut avoir l'idée d'identité culturelle au sein de leur tradition : quelle reconnaissance peut, et doit, faire de l'identité culturelle une théorie de la justice libérale? Le principe de tolérance est-il compatible avec le traitement préférentiel de certaines minorités ethniques? Les demandes de reconnaissance culturelle ne sont-elles pas antilibérales ? Comment réaliser un système démocratique qui garantisse et l'égalité des droits individuels et le respect de la différence identitaire ? Bref, dans quelle mesure doit-on rechercher une nouvelle articulation entre l'individu et le collectif, entre l'identité politique et l'identité culturelle? En ignorant totalement les reproches communautariens, ou en y répondant de manière inappropriée, le libéralisme ne résout nullement le problème posé par le communautarisme, bien au contraire. C'est uniquement en étudiant le contenu de ce phénomène, en pensant la question de l'identité culturelle à l'intérieur même de la pensée libérale ${ }^{5}$ et en tâchant d'y répondre le plus justement possible que les libéraux sauront préserver les acquis humanistes de la tradition libérale, et en éviteront les risques destructeurs pour le lien social. 


\section{NOTES}

1. Robert Nozick, Anarchie, État et utopie [1974], trad. fr. E. d'Auzac de Lamartine, Paris, PUF, 1988.

2. J. Rawls, Théorie de la justice [1971], trad. fr. C. Audard, Paris, PUF, 1995.

3. Ces deux «teams » ont été identifiées par Taylor dans «Cross-Purposes : The LiberalCommunitarian Debate », dans N.-L. Rosenblum, Liberalism and the Moral Life, p. 160. Il faut noter que ces deux fronts sont très hétérogènes ; nombreux sont en effet les penseurs du même courant qui s'opposent sur des questions importantes.

4. M. Walzer, « La critique communautarienne du libéralisme », dans A. Berten, P. Da Silva et H. Pourtois (dir.), Libéraux et communautariens, p. 311-313.

5. C'est l'approche que W. Kymlicka, libéral modéré, tente de mener.

\section{AUTEUR}

\section{CLAIRE DEMESMAY}

claire.demesmay(at)mailbox.tu-dresden.de 